\title{
Variations
}

Variations Revue internationale de théorie critique

15 | 2011

La haine

\section{La haine, cette (violente) muse}

\section{Daniel Veron}

\section{(2) OpenEdition \\ Journals}

Édition électronique

URL : http://journals.openedition.org/variations/99

DOI : 10.4000/variations.99

ISSN : 1968-3960

\section{Éditeur}

Les amis de Variations

\section{Édition imprimée}

Date de publication : 1 mars 2011

\section{Référence électronique}

Daniel Veron, « La haine, cette (violente) muse », Variations [En ligne], 15 | 2011, mis en ligne le 01

février 2012, consulté le 02 mai 2019. URL : http://journals.openedition.org/variations/99 ; DOI :

$10.4000 /$ variations. 99 
Daniel Veron

La haine, cette (violente) muse

« La haine est sainte. Elle est l'indignation des cours forts et puissants, le dédain militant de ceux que fâchent la médiocrité et la sottise. Haïr c'est aimer, c'est sentir son âme chande et généreuse, c'est vivre largement du mépris des choses honteuses et bêtes. La haine soulage, la haine fait justice, la haine grandit.» Émile Zola, Mes haines, 1866

Une "sainte" haine ? Une haine positive, performative, sûrement. Une haine digne. C'est de celle-ci que je voudrais parler ici. De quoi s'agit-il exactement? A peine d'un sentiment, tout au plus une sensation, une impression diffuse d'injustice, un cri.

« Au commencement était le cri. Nous crions. [...] Nous commençons par la négation, la dissonance. La dissonance peut revêtir de multiples formes : un murmure inarticulé de mécontentement, des larmes de frustration, un cri de colère, un rugissement sûr de lui, un trouble profond, un profond désir, une vibration critique. ${ }^{1} »$

Ce cri prend parfois la force de l'évidence, lorsque, dans une dialectique avec la violence brute de l'ordre, il s'exprime comme il peut : dans la violence. Parler de la haine invite à poser la question, centrale s'il en est, des rapports entre politique et violence. Non pas dans un registre moral, de savoir si la violence est ou non légitime, s'il faut voter Guevara ou Gandhi. En posant la

J. Holloway, Changer le monde sans prendre le pouvoir, Syllepse/Lux, Paris/Montréal, 2007 
question en ces termes, nous ne rencontrerions qu'apories. Bien plutôt dans un registre analytique, nous voulons montrer ici que la violence apparaît au contraire comme une dimension constitutive de la politique, en tant que celle-ci est avant tout un rapport émotionnel au monde.

«Si on se jette dehors, avec le diable au corps, c'est qu'on refuse de vivre sans honorer nos morts. "

Hamé, Qui ça étonne encore ?, 2007

Dans le documentaire 365 jours à Clichy-Montfermeil, dont les images sont en parties tournées lors des émeutes de novembre 2005, le réalisateur Ladj Ly, cinéaste membre du collectif Kourtrajmé, filme une voiture de police qui brûle... Un jeune homme présent à l'image commente : "Ça claque sa mère! Ça claque sa mère, ces fils de putes! Je me suis fais tabassé une année, cousin! Je bande! Je bande! Je bande !! Bande d'enculés va!»

Le langage est quelque peu emphatique mais le sentiment est bien là. Et moi, spectateur, je ressens un picotement, une jubilation, l'ivresse des flammes... Pourquoi apprécié-je autant cette scène ?

Les affects sont constitutifs des subjectivités politiques : une haine viscérale de la police peut parfaitement se conjuguer avec une mise en sens de celle-ci, avec un discours qui peut être argumenté par des faits, des données statistiques, des expériences rapportées ou vécues. Dit autrement, ce que l'on appelle la politique - dans son sens noble - est un nœud complexe où affects (la haine, la rage, l'indignation...), pratiques (l'action concrète), et discours (argumentaires, représentations du monde) sont inextricablement enchevêtrés. Le décryptage de ce nœud est au centre de toute réflexivité sur les luttes passées, présentes ou à venir.

$\mathrm{Au}$ cœur de notre propos se trouve je crois l'émeute, en tant que pratique où les émotions 
brutes se donnent à voir. Dans les quartiers populaires en France, c'est précisément le moment où violence et politique se côtoient, se confrontent, dialoguent.

«Contre qui se battent les émeutiers? Contre un ennemi sans visage. Contre ceux qui les nient quotidiennement, les condamnent à l'inexistence sociale et leur réservent un avenir en forme d'impasse. ${ }^{1}$

Faut-il faire remarquer que les explosions de violence les plus importantes et médiatisées de ces dernières années dans les quartiers populaires ont toujours comme déclencheur une « bavure » des forces de l'ordre ? Effet déclencheur de la bavure qui ne se comprend que réinséré dans une interaction quotidienne avec le reste de la société devenu depuis bien longtemps presque exclusivement agonistique. De Zied et Bouna à Moshin et Laramy : «ils» ont tué l'un des leurs l'un des nôtres -, le cri devient destruction. Alain Bertho, anthropologue qui s'attèle à recenser les émeutes dans le monde, montre qu'il s'agit là d'un schéma récurrent de déclenchement des violences collectives $^{2}$.

La violence du mode émeutier a une potentialité politique en ce sens qu'elle combat ouvertement la logique de l'ordre, quand bien même cette violence ne s'appuie pas sur un discours politique construit, ou du moins intelligible dans l'espace public bourgeois, comme dans le cas des émeutes de l'automne 2005 dans les quartiers populaires. À propos de celles-ci Patrick Cingolani analysait : «L'émeute comme expression égarée, comme expression qui n'arrive pas à trouver son sens, est par excellence la manifestation de ce déficit de la politique, à moins qu'elle ne puisse être aussi le moment où cette politique puisse émerger. $»^{3}$

La violence n'est pas un débordement malencontreux, pas plus qu'elle ne serait le fait que de deux ou trois agités. L'ordre policier est lui-même largement engagé dans la violence, seulement

\footnotetext{
C. Bachmann et N. Leguennec, Violences urbaines. Ascension et chute des classes moyennes à travers cinquante ans de politiques de la Ville, Albin Michel, 1997

2 A. Bertho, «Les émeutes dans le monde en 2009 : ethnographie de la colère», Revue internationale et stratégique, n79, 2010 : "Malgré leur fréquent silence, à travers leurs actes euX-mêmes, les émeutiers du XXIe siècle nous parlent abondamment du divorce entre les peuples et les États, de l'effondrement des dispositifs institutionnels et subjectifs de représentation politique et sociale."

3 P. Cingolani, document de travail sur les émeutes de novembre 2005.
} 
il en a, selon la classique formule de Max Weber, le monopole et la légitimité. La politique apparaît liée de façon inextricable à la violence, non pas celle à la fois froide et bestiale des forces de l'ordre, mais bien à la rage, aux tripes.

«Y'a pas de casseurs de vitre, ça existe pas, y'a que des mecs qui veulent exister. »

Nakk, Chanson triste, 2006

La violence porte bel et bien en elle une dimension contestataire. Voici comment Norbert, qui se rendait chaque soir pendant dix jours devant la Sorbonne pour participer aux fameux «débordements» de fin de manifestations lors du mouvement CPE, explique les raisons de son engagement dans le mode émeutier :

«Bah, je vais dire c’est pas vraiment pour casser du keuf, mais c'est un peu ça quoi. Parce que bon, dire "casser du keuf", c'est un peu con, mais bon. C'est pour être sur le front quoi, connaître des sensations. Pour la violence. Le conflit avec les keufs. La guérilla urbaine quoi. C'est ma motivation première.

Après y a un aspect idéologique, ouais. Je déteste ce système, comment il fonctionne. Et pour moi les flics, c'est les représentants... enfin c'est l'État quoi. Ils représentent l'État. Donc quand tu jettes un caillou sur leurs gueules, tu balances un caillou sur l'État. »

Comment prétendre ici faire la part du légitime ou du non légitime, du politique et du non politique, sans porter de fait un jugement moralisant sur l'engagement des acteurs ? Violence, émotions et politique restent inextricables, dans la pratique même de ces derniers. 
« Pendant le CPE, j’étais plus moi-même, j’avais vraiment la rage. Je me reconnaissais plus.

Genre j'avais trop la rage, trop la rage.»

Norbert, artiste

La rage face à l'«État» ou au «système», n'est pas une idée simpliste de jeunes écervelés, mais bien une tentative d'expression de cette haine qui prend littéralement aux tripes. Si le déclencheur de la violence est souvent un acte insupportable de répression, il puise tout autant dans des émotions de haine larvée, haine qui prend sa source dans une expérience affective quotidienne oppressive. Il s'agit de moments où la légitimité du monopole de la violence par les forces de l'État est remise en question car prise en flagrant délit d'injustice. La répression peut ainsi parfois créer une vraie solidarité. K. Ross, dans son étude Mai 68 et ses vies ultérieures, note en effet, à partir de témoignages, une solidarité, qui n'est pas de la pitié ou un vague soutien, mais bien de l'ordre de l'admiration : "Les ouvriers, contrairement aux parents ou aux spectateurs de la classe moyenne, n'étaient pas pris de pitié pour les victimes des gaz lacrymogènes roués de coups, mais plutôt en proie à un sentiment de respect admiratif face à l'action directe menée par les étudiants - une solidarité qui n'était pas de la charité’. ${ }^{1}$ Il en est de même en 1986, pendant le mouvement contre la réforme Devacquet, entre les étudiants qui trouvent les lycéens trop excités et incontrôlables, mais ne les respectent pas moins pour leur courage, leur motivation et leur efficacité dans la prise de possession de la rue. ${ }^{2}$ C'est sans doute cette efficacité qui a valu une telle répression sur les mobilisations lycéennes lors du mouvement d'octobre 2010, de Nanterre à Montreuil, en passant par la Place Bellecour à Lyon³.

A chaque fois, le moteur est bien une rage, une haine, une colère, d'où peut effectivement émerger la violence autant que la parole politique, ou les deux. Mais ce sont des sentiments dignes -

\footnotetext{
K. Ross, Mai 68 et ses vies ultérieures, , Bruxelles, Editions Complexe, 2005

G. NAMER, Mémoire et projet du mouvement lycéen-étudiant de 1986-1988, L'Harmattan, Paris, 1990

Sur les événements du 21 octobre 2010 Place Bellecour à Lyon, voir les témoignages rassemblés sur http://rebellyon.info/Temoignages-sur-la-prison.html
} 
et sans doute est-ce pour cela que Zola parle de « sainte haine ».

L'expérience des sans-papiers en France $^{1}$ montre également le caractère central de l'expérience de l'oppression dans l'engagement politique.

Octobre 2005, à la station Château d'eau, alors qu'une rafle est en cours, la situation va vite dégénérer et virer à l'émeute. Sylvain Georges, cinéaste, est présent sur les lieux et filme toute la scène $^{2}$. Au début, ils ne sont que quelques-uns à commencer à protester, puis de plus en plus nombreux. Des poubelles sont jetées en travers de la rue, sur les fourgons de police ; divers projectiles volent et viennent éclater sur les boucliers des forces de l'ordre ; les manifestants scandent : "Libérez les sans-papiers! ». Révolte soudaine et spontanée face à une pression sourde et permanente. La rafle sera finalement stoppée, et même si plusieurs dizaines de sans papiers sont arrêtés, les participants semblent satisfaits d'avoir pu faire fuir la police, et éviter qu'il n'y ait plus d'interpellations. Face à la caméra de S. Georges, un sans papiers explique :

« Les gens, ils ont ras-le-bol ! C'est inhumain ce que pratique l'administration ! C'est inacceptable ! Chaque fois que j'entends les politiques parler d'humanité, de trucs comme ça, j'ai... j'ai envie de pleurer ! Alors c'est pour ça, je suis prêt à faire tout! A faire la grève de la faim, à révolter, à faire n'importe quoi pour avoir... la dignité ! C'est pour ça, je... je peux plus continuer à vivre comme ça ! Impossible... Impossible. »

Il est étonnant de voir que les analyses portant sur les mouvements sociaux n'abordent quasiment jamais ce qui pourtant en est le moteur : les tripes, la rage, la haine. Dimension en effet rarement traitée par la sociologie de l'action collective, la question des affects n'en est pas moins centrale dans le déroulement de l'action collective. Parce que l'exclusion est source de souffrances, elle active de fait la dimension affective.

\footnotetext{
Sur le sujet voir l'ouvrage collectif signé Iana MAR,Travailleurs, vos papiers !, Libertalia, Paris, 2011.
}

2 S. Georges, N'entre pas sans violence dans la nuit, Noir Productions, 2007 
« C'est quoi ces gens-là? Ils reconnaissent même pas le vécu, tout ce qu'on a vécu en France.

C'est très dur. »

Dembele, « Oublié de St-Paul » (parlant de la préfecture).

Face à une violence sociale, les ajustements des individus sont multiples. Le développement de pathologies somatiques en est un, sans doute extrêmement répandu, mais difficilement mesurable étant donné que les sans-papiers ont difficilement accès aux soins. Didier Fassin, sociologue et médecin, raconte ainsi, dans l'ouvrage collectif Les lois de l'inhospitalité paru en 1997, le cas hélas fréquent d'une victime de la double peine, qui, apprenant la confirmation de son interdiction de territoire à sa sortie de prison, entre en grave dépression, développe un ulcère gastrique et perd ses cheveux, «somatisations que l'on peut considérer comme la seule manière d'exprimer une souffrance indicible. ${ }^{1} \gg$

Les affects induits par cette violence peuvent parfois mener, et sans doute plus souvent qu'on ne le croit, à retourner celle-ci contre soi : des simples mutilations, jusqu'à la plus extrême des formes de violence sur soi, le suicide.

« Moi ça fait... je suis là ça fait 17 ans. [...] J'ai presque passé toute ma vie en souffrance.

[...] Tu vois, y'a des gens qui se suicident... Ils ont raison! Parce que... tu sais plus quoi faire !! La seule façon c'est de te suicider comme ça tu... enfin tu seras tranquille quoi. » Mamadou, «Oublié de St-Paul»

Parce qu'il est au cœur du dispositif de gestion policière de l'immigration, le centre de rétention administrative, ou CRA, ne peut que rendre encore plus radicale les émotions et les affects en jeu dans les rapports que les sans-papiers peuvent avoir avec le système qui les réprime : pathologies, tentatives de suicides, mutilations sont des événements du quotidien. Mais ces affects

\footnotetext{
D. Fassin, «La santé en souffrance», in D. Fassin, A. Maurice, C. Quiminal (dir.), Les lois de l'inhospitalité, les politiques de l'immigration à l'épreuve des sans-papiers, La Découverte, Paris, 1997.
} 
peuvent trouver leur expression dans une autre forme : la révolte, individuelle ou collective.

«Brûle, Babylon, brûle! Tu ne mettras pas mes mots en cellule!»

Djamal, Fou à Nier II, 1998

L'ouvrage Feu au centre de rétention ${ }^{1}$ est précieux pour tenter de saisir ce que peut être cette expérience d'enfermement. Pendant plusieurs mois, des militants ont appelé les cabines téléphoniques à l'intérieur du centre. Nouant peu à peu une relation de confiance, ils ont recueillis des témoignages du quotidien des sans-papiers détenus au CRA de Vincennes. Les témoignages s'étalent sur 6 mois, de janvier à juin 2008, jusqu'au jour où ce centre fut incendié, suite à la mort d'un détenu tunisien, Salem Essouli.

Dans les centres, le refus est continuel, par-delà le renouvellement de la population, mais varie dans son intensité et ses formes, et passe bien souvent par le retournement de la violence sur soi. Ca le désespoir est aussi une réponse à la violence de la logique policière : les mutilations (veines tailladés, ingestion de lames de rasoirs, pendaisons...), tout est bon pour échapper à l'expulsion, d'autant que souvent c'est la mort (si ce n'est physique, au moins sociale) qui les attend de toute façon, si jamais ils sont effectivement reconduits.

"Pour refuser d'embarquer, un mec a eu une idée incroyable. Il s'est chié dessus. Il s'est tout étalé sur lui. Ils n'ont pas pu l'expulser. Ils l'ont ramené au centre. Le lendemain, ils sont venus le chercher. Ils l'ont attaché avec du scotch et ils l'ont enroulé dans du film plastique. Ils l'ont pris et ils l'ont expulsé comme ça. »*2

Feu au centre de rétention, des sans-papiers témoignent (janvier à juin 2008), Éditions Libertalia, Paris, 2008.

2 Les citations marquée d'un* sont issues de l'ouvrage Feu au centre de rétention, op. cit. 
Mais les refus prennent heureusement d'autres formes. Souvent autour de la nourriture, d'une télé éteinte sans raison ${ }^{1}$, les protestations fusent, parfois jusque dans des altercations violentes où les sans-papiers ont rarement l'avantage (généralement celui qui est jugé avoir déclenché l'événement est passé à tabac). Mais chaque fois c'est l'ordre oppressant qui est grippé, lorsque la révolte contre celui-ci s'exprime.

« Les refus de comptage, je dirais que c'est presque tous les jours. Parfois on refuse un peu. Parfois on refuse beaucoup. »*

Le refus du contrôle, comme refus de l'emprise totale de l'ordre, participe de ce que, Foucault nomme une "échappée" propre à ceux qui font l'objet d'oppression. Si cette fuite ne trouve parfois que le suicide et l'automutilation, elle peut réussir à trouver une force toute particulière dans une lutte collective.

« On a commencé la grève de la faim ce midi. Personne n'est allé manger. Six policiers sont allés voir les Chinois pour leur dire de manger. Ils ont refusé. Nous nous sommes tous regardés et on a rigolé.»*

La grève de la faim est ainsi une des formes qui revient régulièrement dans le centre de rétention de Vincennes. Sur les six mois que couvre l'ouvrage, les témoignages font l'état d'au moins une dizaine de tentatives. Chaque fois, elle ne dure que peu de temps, deux trois jours, tant l'organisation collective est rendue difficile par la pression policière, mais aussi par le turnover des détenus, sans compter la barrière de la langue entre les différentes communautés.

Multiples sont également les tentatives d'incendie. Les témoignages relatent en effet de nombreux départs de feu. Jusqu'à ce 22 juin 2008. La veille, un retenu tunisien, Salem Essouli, à

\footnotetext{
Comme cette même nuit où la police fera pour la première fois en France usage d'un Taser. Télé éteinte sans raison, le ton monte, les retenus refusent de remonter dans les chambres. Les CRS sont envoyés. S'ensuit un tabassage "dans l'escalier, dans les couloirs, dans les chambres". Alors que le calme est revenu depuis peu, tous les détenus sont réveillés et déplacés dans la cour. Les chambres sont fouillées. Les détenus retrouveront "un Coran déchiré et piétiné, des fils de chargeurs coupés".
} 
cause d'un défaut de soin comme il en arrive fréquemment, est mort. S'ensuivra une nuit de chaos au bout de laquelle le centre partira en fumée. Le 9 avril, prémonitoire, un détenu déclarait :

« Il faut penser la lutte autrement. Les gens et les flics se foutent de la grève de la faim. Ils se foutent des sans-papiers. Ils s'en foutent si on crève. Les gens bouffent des lames de rasoir tous les jours et on n'entend pas parler d'eux. Les petits trucs qu'on fait ne valent pas le coup. Il faut que ça pète pour que les gens s'intéressent à nous. \**

L'incendie du centre de rétention de Vincennes a largement participé à la construction d'une visibilité dans l'espace public et à une publicisation du traitement réservé aux étrangers enfermés dans ces centres. Seulement, le sens d'une telle action est difficilement contrôlable une fois jetée dans le champ de bataille médiatique. A contrario, la grève de la faim, qui met le corps et la vie ellemême dans la balance, conférant à l'engagement ainsi un caractère hautement critique, est forte du point de vue du sens ${ }^{1}$ mais manque de visibilité publique et reste souvent invisible dans l'espace clos du centre.

«Quand vous explosez à bon droit, sous le poids des injustices qui vous accablent, ils se servent de la presse pour vous présenter comme des vandales. »

Malcolm X

Si la violence passe aujourd'hui pour une dimension illégitime du rapport à la politique, qui renverrait à la part animale, barbare des individus, elle a au moins clairement le mérite d'imposer une présence dans l'espace public bourgeois.

Ce fut le cas de l'incendie du CRA de Vincennes, mais on retrouve la même logique dans un

\footnotetext{
1 Voir l'analyse de P. Cingolani, La République..., op. cit., qui montre que, loin de poser le sans-papiers en victime, la
} grève de la faim permet la "sortie du néant social" dans lequel reste confiné la figure du clandestin. 
tout autre contexte, le conflit des salariés de Continental :

- David Pujadas : « Bonsoir Xavier Mathieu, vous êtes le délégué CGT de Continental à Clairoix. On comprend bien sûr votre désarroi, mais est-ce que ça ne va pas trop loin ? Est-ce que vous regrettez ces violences? »

- Xavier Mathieu : «Vous plaisantez j’espère ? On regrette rien... »

- DP : « Je vous pose la question. »

- XM : « Non, non, attendez. Qu'est-ce que vous voulez qu'on regrette? Quoi ? Quelques carreaux cassés, quelques ordinateurs à côté des milliers de vies brisées ? Ca représente quoi ? Il faut arrêter là, il faut arrêter. »

- DP : « Pour vous la fin justifie les moyens. »

- XM : «Attendez, la fin. On est à 28 jours de la fin, monsieur. On est en train de nous expliquer que dans 28 jours [images de saccage reprises en parallèle] le plan social sera bouclé et on va aller à la rue. Oui, oui, je ne regrette rien. Personne ne regrette rien ici parce que vous avez vu, vous avez pas vu des casseurs, vous avez vu des gens en colère, des gens déterminés, des gens qui veulent pas aller se faire démonter, crever. On ne veut pas crever. On ira jusqu'au bout de notre bagarre. On a tenu cinq semaines. Pendant cinq semaines j'ai réussi, on a retenu, on a réussi à retenir les gens. C'est fini, les gens n'en veulent plus. Le gouvernement nous a fait des promesses. Il s'est engagé à réunir une tripartite depuis le début, dans les trois jours. Ca fait une semaine que ça dure. Depuis on se rend compte...»

- DP : « Xavier Mathieu, on entend votre colère, mais est-ce que vous lancez un appel au calme ce soir ?»

- XM : « Je lance rien du tout. J'ai pas d'appel au calme à lancer. Les gens sont en colère et la colère il faut qu'elle s'exprime. Il y a un proverbe des dernières manifestations qui dit qui sème la misère récolte la colère. C'est ce qu'ils ont aujourd'hui. Il y a plus de 1000 familles qui vont être à la rue qui vont crever dans 23 mois avec plus rien, qui vont être obligées de vendre leur baraque. Il 
faut que tous vous compreniez ça. On ne veut pas crever... »

Ici, affects - cette colère -, pratiques - saccage de la préfecture -, discours - argumentation de la légitimité de leur action et sa contextualisation -, sont des dimensions inextricables de ce « moment politique ${ }^{2} »$.

De la même manière, les ouvriers, victimes des récents plans sociaux, ont inauguré (ou plus exactement remis au goût du jour) de nouvelles tactiques d'actions, souvent montrées du doigt comme violentes, mais bel et bien efficaces. La rage suscitée par le mépris patronal et le caractère hautement critique de leur situation les amène à séquestrer leurs dirigeants. Ils ne s'engagent pour autant dans la violence physique, qui aurait pu être une réponse émotive, mais sans doute une mauvaise tactique eu égard aux contraintes de justification publique. Au contraire, par la rationalisation politique de leurs affects, et la justesse de leur tactique, ils ont forcé l'interlocution afin de faire entendre leurs raisons.

C'est bien dans une retraduction des affects en actes et en discours que tactiques et argumentaires politiques prennent leur force. Mais une telle retraduction n'est en rien donnée ni garantie.

L'émergence d'une parole qui confèrera un sens aux actions qui font éruption dans l'espace public est en effet cruciale, afin de rendre intelligible et convaincre de la justesse de ses actions. Mais bien souvent cette parole, à défaut d'apparaitre comme légitime dans l'espace public bourgeois - qui n'y entend que du bruit -, circule dans des espaces publics périphériques, ce que Negt appelle espace public oppositionnel. Le film de Ladj Ly, mentionné plus haut, en fournit un fragment.

—Et vous croyez que ça va changer quelque chose en jetant des pierres et tout?

$-S i, s i$

21 avril 2009, 20h de France 2. J'emprunte la transcription à Acrimed : http://www.acrimed.org/article3132.html

2 J. Rancière, Moments politiques, La Fabrique, Paris, 2009. 
— Si ça change rien, ça nous fait du bien de... mais on espère que ça va changer quelque chose.

— Et là donc qu'est-ce qu'il se passe? Vous les attendez pour leur jeter des pierres

- Parce qu'on parle on parle, on n'est pas entendu. Le seul moyen de se faire entendre c'est avec des pierres.

— Mais entendre... donc vous avez des choses à dire alors.

- On a beaucoup de choses à dire!

— Bah alors allez-y dites le. Qu'est-ce que vous avez à dire?

— On a à dire quoi ? On existe ! On est là !

- On est pas des chiens.

- Bah ouais!

— Et tu crois que c'est sans flics que les choses vont s'arranger?

- C'est pas sans flics. Mais avec des flics qui flambent, ça le fait pas.

— Allez rentrez chez vous là. Ça sert à rien de niquer les keufs. Ça va rien changer. Allez, allez.

Sans doute «niquer les keufs» ne changera pas le monde. Sans doute cela marque-t-il un manque d'organisation, de stratégie, de structuration politique ou que sais-je encore. Et sans doute cela est-il regrettable. Mais ils est totalement compréhensible qu'il en soit ainsi, que ces jeunes veuillent «niquer les keufs». Ont-ils raison, ont-ils tort ? Là n'est pas tellement la question. Le fait est qu'un tort est exprimé : il n'est pas légitime d'être humilié par la police ("les flics flambent").

Quels autres moyens ont-ils alors pour exprimer ce grief ("le seul moyen de se faire entendre c'est de jeter des pierres") ?

« Derrière chaque caisse qui crame se cache une voix qu'on entend à peine. "

Fabe, On m'a dit, 2000 
Sophie Wahnich ${ }^{1}$ nous rappelle très justement combien l'idée que la question politique puisse faire l'économie de l'engagement des corps, de la violence, du sang, procède d'un mythe moderne. S'intéressant à la période de la Terreur, elle montre que la violence révolutionnaire aujourd'hui unanimement condamnée relève d'une volonté, à toute épreuve, de protéger la révolution contre toute menace à la souveraineté du peuple. Cette souveraineté, si durement acquise, est ainsi élevée au rang du sacré. La "vengeance", face à l'ennemi qui menace la révolution, implique des "transactions sacrées", où le sacrifice est exigé.

"L'effroi, le dégô̂t, la terreur et l'enthousiasme sont les émotions qui signent de l'expérience de cette bordure [du politique avec le sacré], là où la révolution et ses acteurs peuvent sombrer dans le néant. [...] Nul n'est vraiment à l'abri d'une transaction sacrée où la fondation des valeurs exige la mort des hommes, où il faut s'engager corps et âme, où chacun peut périr d'effroi ou être gagné par le dégoût. »"

À l'expérience du tort, constitutive de la politique, il n'existe aucune réponse évidente. Prise de parole, violence ou profond dégoût sont diverses formes de réactions face au tort ; et si elles sont parfois contradictoires, un même individu, un même collectif, peut pourtant éprouver, exprimer ces différents modes. Au même noyau d'injustice émerge la politique, la violence ou le dégoût, ou les trois à la fois.

«L'absence d'espaces publiques où la parole populaire pourrait se frayer un chemin, être entendue et répercutée sous formes de lois apaisantes, a partie liée avec le surgissement de la violence $»^{3}$, mais aussi de toutes ces formes de dégoût ou d'apathie, dont le degré premier est l'abstentionnisme. Mais cette conscience aiguë du caractère vain de la lutte contre l'injustice, peut prendre des formes extrêmes. P. Cingolani cite ainsi les propos de Kaled Kelkal, qui sera impliqué dans les attentats du RER en 1995 : «Moi j'étais intéressé à la politique, j'ai suivi la politique,

S. WAHNICH, La liberté ou la mort, essai sur la terreur et le terrorisme, Paris, La fabrique, 2003.

Idem.

Idem. 
mais c'est de la connerie $»^{1}$.

Les éléments qui déterminent les canaux d'expression que suivront les émotions sont multiples, et toutes les occurrences politiques ont leurs singularités et leurs particularités. Entre émeutes, mouvements sociaux organisés, retournement de la violence sur soi, ou dépolitisation collective, voire violence dite terroriste. Des émotions, la politique tire sa force ; tout autant qu'elle menace de s'y épuiser.

Une chose toutefois semble sûre : parce que c'est précisément sur les affects que porte le poids de l'ordre, violence et haine - par nécessité dialectique - marqueront les temps à venir.

Daniel Veron

P. Cingolani, document de travail sur les émeutes, op. cit. 